\title{
100 nm-Gap Fingers Dielectrophoresis Functionalized MOX Gas Sensor Array for Low Temperature VOCs Detection ${ }^{\dagger}$
}

\author{
Luca Francioso *, Pasquale Creti, Maria Concetta Martucci, Simonetta Capone, Antonietta Taurino, \\ Pietro Siciliano and Chiara De Pascali \\ National Research Council of Italy, Institute for Microelectronics and Microsystems,73100 Lecce, Italy; \\ creti@le.imm.cnr.it (P.C.); martucci@le.imm.cnr.it (M.C.M.); simonetta.capone@le.imm.cnr.it (S.C.); \\ antonietta.taurino@le.imm.cnr.it (A.T.); pietro.siciliano@le.imm.cnr.it (P.S.); depascali@le.imm.cnr.it (C.D.P.) \\ * Correspondence: luca.francioso@le.imm.cnr.it; Tel.: +39-832-422-525 \\ † Presented at the Eurosensors 2018 Conference, Graz, Austria, 9-12 September 2018.
}

Published: 13 November 2018

\begin{abstract}
Present work reports the fabrication process and functional gas sensing tests of a $100 \mathrm{~nm}-$ gap fingers DiElectroPhoresis (DEP) functionalized MOX (Metal OXide) gas sensor array for VOCs detection at low temperature. The Internet of Things (IoT) scenario applications of the chemical sensing-enabled mobiles or connected devices are many ranging from indoor air quality to novel breath analyser for personal healthcare monitoring. However, the commercial MOX gas sensors operate at moderate temperatures $\left(200-400{ }^{\circ} \mathrm{C}\right)$ [1], and this limits the mobile and wearable gadgets market penetration. Nanogap devices may represent the alternative devices with enhanced sensitivity even at low or room temperature. A nanogap electrodes MOX gas sensor array functionalized with $5 \mathrm{~nm}$ average size $\mathrm{SnO}_{2}$ nanocrystals with positive dielectrophoresis technique is presented. The single sensor active area is $4 \times 4 \mu \mathrm{m}^{2}$. The devices exhibited about 1 order of magnitude response at $100{ }^{\circ} \mathrm{C}$ to $150 \mathrm{ppm}$ of acetone.
\end{abstract}

Keywords: nanogap sensor; metal oxide sensor; nanofabrication; electron beam lithography; microelectronic fabrication; low temperature gas sensing

\section{Introduction}

In the recent years, the IoT revolution offers new opportunities to explore specific applications with the support of mobile phones and wireless connected devices. However, the MOX sensors (e.g., $\mathrm{SnO}_{2}$ thick or thin film devices) operate at $200{ }^{\circ} \mathrm{C}-400{ }^{\circ} \mathrm{C}$ [1], and several methods have been developed to lower the operative temperature, such as noble metal doping or UV-VIS light activation [2]. Considering the large market interest in gas sensing technologies for mobile phones and portable devices, detection at low power consumption and low concentrations are driving challenges for nanogap and microfabricated devices [3-7]. Up to now, there are few studies on the detection of volatile organic compounds (VOCs) with nanogap devices for air quality and personalized health monitoring. To this aim, acetone represents one of the most common VOCs widely used in the industry and it is a selective breath marker for diabetes monitoring; thus a cheap detection method, like ultra-low power MOX gas sensors may be strategic in the follow-up care of diabetic disease.

Present work reports the fabrication process and functional gas sensing tests of a $100 \mathrm{~nm}$-gap fingers DiElectroPhoresis (DEP) functionalized MOX (Metal OXide) gas sensor array for VOCs detection at low temperature. The Internet of Things (IoT) applications of the chemical sensingenabled mobiles or connected devices are many and have been attracting a growing attention from retail market players. However, the commercial MOX gas sensors operate at moderate temperatures 
$\left(200-400{ }^{\circ} \mathrm{C}\right)[1]$, and this aspect limited the mobile and wearable gadgets market penetration. Nanogap devices may represent the alternative devices with enhanced sensitivity even at low or room temperature. A nanogap electrodes MOX gas sensor array functionalized with $5 \mathrm{~nm}$ average diameter $\mathrm{SnO}_{2}$ nanocrystals with positive dielectrophoresis technique was fabricated. The single sensor active area is $4 \times 4 \mu^{2}$.

\section{Materials and Methods}

Present work proposes a complete MEMS fabrication process for a metal oxide gas (MOX) sensor array (4 devices) on Si substrate realized with e-beam lithography process for Ti/Pt $(5 / 85 \mathrm{~nm})$ interdigitated electrode; gold micrometric electrode for bonding pads were realized on platinum contact for final package. The single sensor active area is about $4 \times 4 \mu \mathrm{m}^{2}$, while the area of whole 4sensor array is $50 \times 50 \mu \mathrm{m}^{2}$ (Figure 1). The nanogap sensors were functionalized with $\mathrm{SnO}_{2}$ nanocrystals (NCs) ( $5 \mathrm{~nm}$ average diameter) synthesized by sol-gel precipitation and dispersed in pure hexane with optimized NCs concentration; the DEP (DielEctroPhoresis) technique was adopted to increase the concentration of tin oxide nanoparticles between the fingers electrodes applying different AC electric field ( 8.0 and $\left.10.0 \mathrm{~V}_{\mathrm{pp}}, 100 \mathrm{KHz}\right)$, followed by $1 \mu \mathrm{L}$ NCs solution dropping onto fingers area up to complete evaporation of solvent.
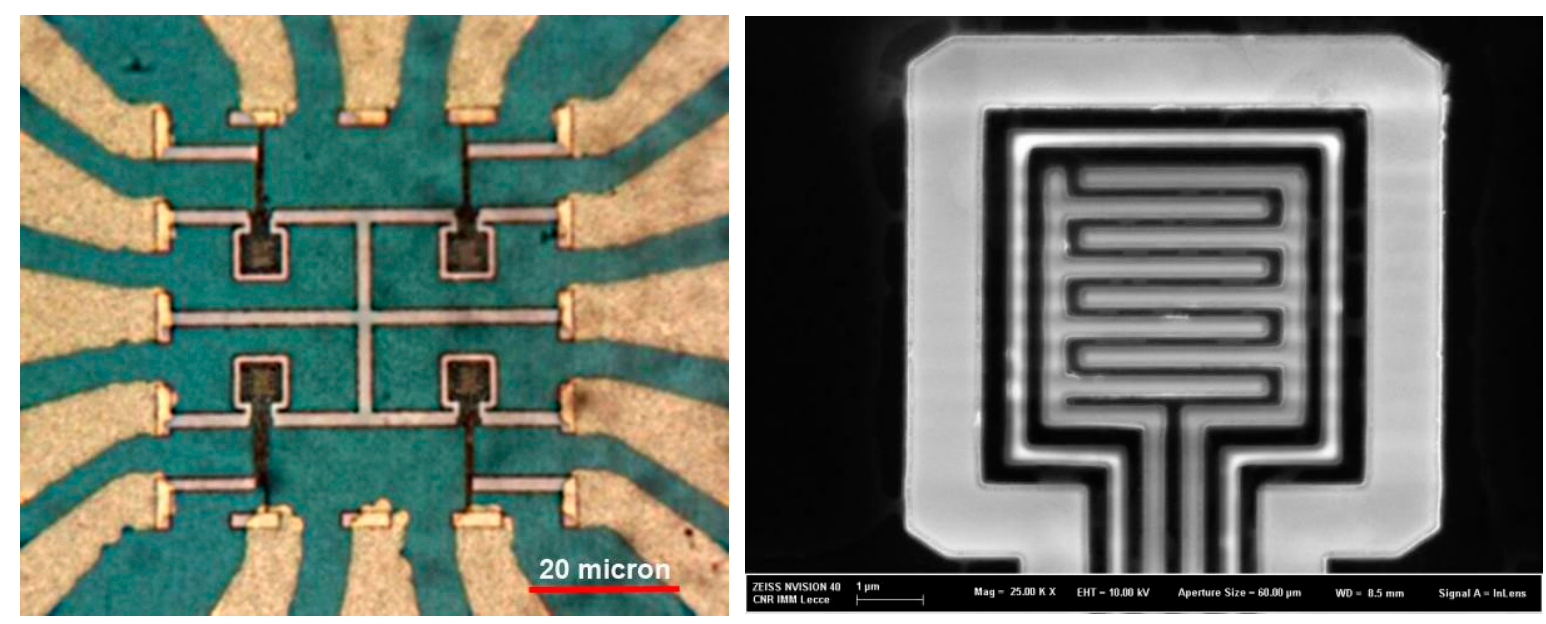

Figure 1. Fabricated MOX sensor array onto silicon substrate (left), SEM image of the single nanogap device with embedded heater and thermometer (right).

\section{Results and Discussion}

Different sensors with different solution concentrations and applied DEP fields were produced and measured at different acetone concentration in dry air and low temperatures (up to $100{ }^{\circ} \mathrm{C}$ ). A preliminary investigation about the current-voltage characteristic of the devices was performed, in order to assess to nano-fingers functionality and lack of electrical shorts among them. The SEM investigation confirmed the localization of nanoparticles clusters between the platinum fingers, as showed on Figure 1, right.

Figure 2 shows the array configuration of the fabricated device: 4 independent sensors were realized, an embedded heater with common ground contact and a test thermometer was located in the bottom-left position; the validation of the mixed fabrication process (e-beam tool for nanometric features and optical lithography for micrometric pads) will open the possibility to fabricate the devices on suspended dielectric hotplates, lowering the power consumption below $1 \mu \mathrm{W}$. 


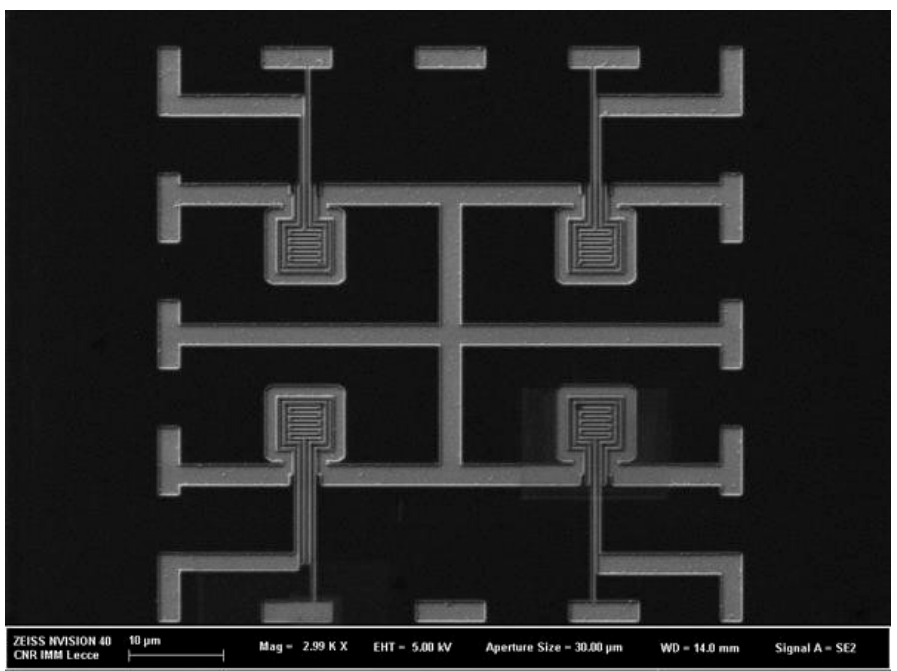

Figure 2. SEM image of an array of 4 nanogap sensor devices with embedded heater and thermometer.

Best response sensor (ID8-1, $8 \mathrm{~V}_{\mathrm{pp}}, 100 \mathrm{kHz}$ AC field) exhibits about 1 order of magnitude response at $100{ }^{\circ} \mathrm{C}$ to $150 \mathrm{ppm}$ of acetone, while the devices with fastest response times are the ID13-1 and ID13-3 (10V $\mathrm{V}_{\mathrm{pp}}, 100 \mathrm{kHz}$ AC field) (Figure 3).The fabricated nanogap devices allow a lower temperature operation of the gas sensors with reasonable response time; work is in progress to compare the responses of micrometric fingers gas sensors with the nanogap-based devices.
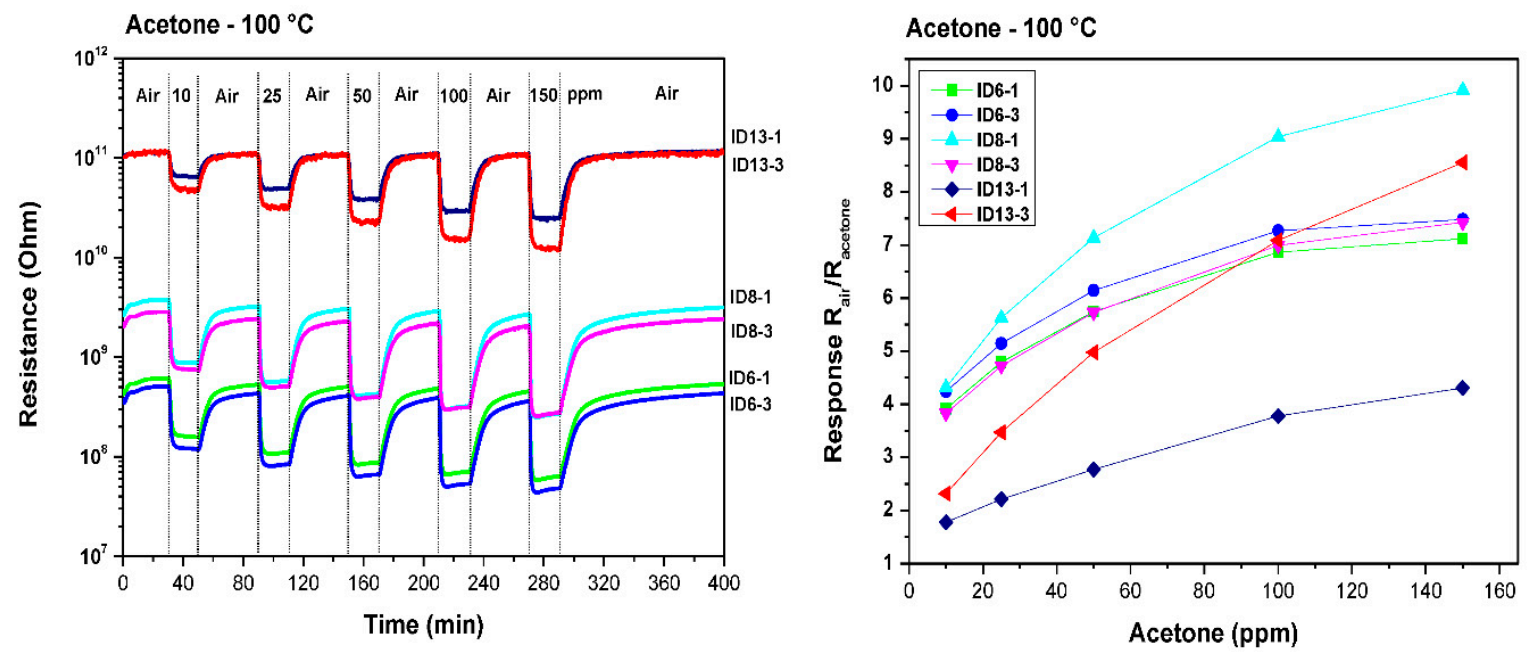

Figure 3. Dynamic profiles and resistance ratio responses of six different nanogap sensors at $100{ }^{\circ} \mathrm{C}$ to different acetone concentrations in dry air.

Conflicts of Interest: The authors declare no conflict of interest.

\section{References}

1. Zhao, Q.; Ju, D.; Song, X.; Deng, X.; Ding, M.; Xu, X.; Zeng, H. , Polyhedral Zn2SnO4: Synthesis, enhanced gas sensing and photocatalytic performance, Sens. Actuators B 2016, 229, 627-634.

2. Zheng, Z.Q.; Yao, J.D.; Wang, B.; Yang, G.W. Light-controlling, flexible and transparent ethanol gas sensor based on $\mathrm{ZnO}$ nanoparticles for wearable devices. Sci. Rep. 2015, 5, 11070, doi:10.1038/srep11070.

3. Menumerov, E.; Marks, B.A.; Dikin, D.A.; Lee, F.X.; Winslow, R.D.; Guru, S.; Sil, D.; Borguet, E.; Hutapea, P.; Hughes, R.A.; et al. Sensing Hydrogen Gas from Atmospheric Pressure to a Hundred Parts per Million with Nanogaps Fabricated Using a Single-Step Bending Deformation. ACS Sens. 2016, 1, 73-80.

4. Francioso, L.; De Pascali, C.; Bartali, R.; Morganti, E.; Lorenzelli, L.; Siciliano, P.; Laidani, N. PDMS/Kapton Interface Plasma Treatment Effects on the Polymeric Package for a Wearable Thermoelectric Generator. ACS Appl. Mater. Interfaces 2013, 5, 6586-6590. 
5. Adami, A.; Lorenzelli, L.; Guarnieri, V.; Francioso, L.; Forleo, A.; Agnusdei, G.; Taurino, A.M.; Zen, M.; Siciliano, P.A. Sens. Actuators B Chem. 2006, 117, 115-122.

6. Epifani, M.; Francioso, L.; Siciliano, P.; Helwig, A.; Mueller, G.; Díaz, R.; Arbiol, J.; Morante, J.R. Sens. Actuators B 2007, 124, 217-226.

7. Presicce, D.S.; Francioso, L.; Epifani, M.; Siciliano, P.; Ficarella, A. Sens. Actuators B 2005, 111-112, 5257.

(C) 2018 by the authors; Licensee MDPI, Basel, Switzerland. This article is an open access article distributed under the terms and conditions of the Creative Commons Attribution (CC BY) license (http://creativecommons.org/licenses/by/4.0/). 